Research Article

\title{
Topic Relevance of Public Health Emergencies Influence on Internet Public Opinion Resonance: Simulation Based on Langevin's Equation
}

\author{
Quan Cheng, Yan-gang Zhang, and Yi-quan Li iD \\ School of Economics and Management, Fuzhou University, Fuzhou, Fujian 350116, China \\ Correspondence should be addressed to Yi-quan Li; xgb@fzu.edu.cn
}

Received 1 August 2020; Revised 10 December 2020; Accepted 21 December 2020; Published 20 January 2021

Academic Editor: Haipeng Peng

Copyright (c) 2021 Quan Cheng et al. This is an open access article distributed under the Creative Commons Attribution License, which permits unrestricted use, distribution, and reproduction in any medium, provided the original work is properly cited.

\begin{abstract}
Public health emergencies occurred frequently, which usually result in the negative Internet public opinion events. In the complex network information ecological environment, multiple public opinion events may be aggregated to generate public opinion resonance due to the topic category, the mutual correlation of the subject involved, and the compound accumulation of specific emotions. In order to reveal the phenomenon and regulations of the public opinion resonance, we firstly analyze the influence factors of the Internet public opinion events in the public health emergencies. Then, based on Langevin's equation, we propose the Internet public opinion stochastic resonance model considering the topic relevance. Furthermore, three exact public health emergencies in China are provided to reveal the regulations of evoked events "revival" caused by original events. We observe that the Langevin stochastic resonance model considering topic relevance can effectively reveal the resonance phenomenon of Internet public opinion caused by public health emergencies. For the original model without considering the topic relevance, the new model is more sensitive. Meanwhile, it is found that the degree of topic relevance between public health emergencies has a significant positive correlation with the intensity of Internet public opinion resonance.
\end{abstract}

\section{Introduction}

In recent years, public health emergencies occur frequently and bring various kinds of Internet public opinion events. Due to the complex network information ecological environment interweaving with amounts of fake news, the resulting negative Internet public opinion arose and spread rapidly, which had caused great obstacles for public health departments to effectively identify, judge, and formulate effective response strategies. The Internet public opinion events of public health emergencies are especially prominent in the social media environment. Since social network attracts more and more netizens to participate by virtue of its universality, interactivity, group, autonomy, and other characteristics, these events are increasingly tending to the complex situation of correlation and serialization [1]. That is, the single Internet public opinion event may be aggregated with the historical public opinion events due to the topic category, the mutual correlation of the subject involved, and the compound accumulation of specific emotions. Accordingly, the public opinion cluster will be formed and the phenomenon of public opinion co-vibration (i.e., public opinion resonance) is generated.

Internet public opinion resonance between emergencies refers to the "resurgence" of public opinion of historical events (called evoked events) caused by public opinions of recent events (called original events). In general, the time interval between events is relatively long, and the degree of public opinion deviation is low due to the "long-term memory" left by history. Take example for COVID-19, the Internet public opinion has been rapidly polarized when Wuhan was sealed in January 23, 2020. COVID-19 is a new unknown virus and belongs to the same cluster with SARS coronavirus (SARS) virus. Furthermore, COVID-19 develops the similar serious diseases as SARS and Middle East respiratory syndrome coronavirus (MERS COV). So, the SARS epidemic event and the MERS COV epidemic event 
were reactivated and have triggered the public initial memory about COVID-19. These related public health emergencies continuously interact, derive, and aggregate to produce public opinion resonance event clusters, which have caused huge social impact in a short period of time. The public opinion resonance event clusters can be revealed by the Baidu index, as shown Figure 1. The Baidu index of SARS quickly arose from 760 in January 19, 2020, to 923683 in January 25, 2020. During this period, the Baidu index of MERS COV quickly arose from 1196 to 11900 . Since the World Health Organization named COVID-19 as new coronavirus pneumonia (NCP) in February 11, 2020, the Baidu index of the "novel coronavirus" keyword quickly reached the first peak of 22336 in February 13, 2020, and also triggered the MERS COV keyword Baidu index of the other high peak of 9261, which generated the resonance of the two Internet public opinion events.

In the social media environment, the negative effects caused by the public opinion superposition resonance of the topic-related events in public health emergencies are more significant than a single event. Furthermore, small changes may trigger the long-term and colossal chain reaction of the public opinion events cluster. Hence, in the frequent topic-related and serial public health emergencies, the characteristics and the regulations of the public opinion resonance should be studied, which assists the public health department to accurately identify the public health information and build a public opinion monitoring and early warning mechanism for public health emergencies.

With these motivations, we address the following questions: (1) what factors influence the public opinion resonance in public health emergencies? (2) How do we accurately describe the regulations of the Internet public opinion resonance? We first extend the existing influence factors, including the topic factors, the regional factors, the theme similarity, and the external emotional intensity of public opinion events. Based on Langevin's equation, we next develop an Internet public opinion stochastic resonance model considering the topic relevance of the multiple public health emergencies. Finally, three exact cases are provided to reveal the regulations of evoked events "revival" caused by original events.

The rest of this paper is organized as follows. Section 2 briefly reviews literature from three aspects. In Section 3, we firstly analyze the influence factors of the public opinion resonance. Then, we develop an Internet public opinion stochastic resonance model based on Langevin's equation. Section 4 discuses three exact cases from the public health emergencies in China. Finally, Section 5 concludes the paper with future research directions.

\section{Literature Review}

Public opinion resonance mainly reveals the correlation or the interaction of multiple public opinion events. In this section, we review the literature from the following aspects: influence factors, the model, and empirical research.
Due to the compound accumulation of specific public opinion and netizens' emotions, there exist kinds of influence factors, which result in different public opinion events and the according public opinion resonances. In the present literature, the influence factors of public opinion resonance mainly focus on three following types: in practices, Internet public opinion events on longer occur in isolation. The events usually are interrupted or persist with the same label, the same subject, the same category, or the same segment sequence events. Media agenda-setting, general preferences of netizens, and other factors promote public opinion events to "mass production" and "similar reproduction" in the short term $[2,3]$. The association superposition will serialize and centralize issues and then generate new hotspots. The superposition mainly focuses on the dimensions of "time," "space," and "symbol," including not only the information superposition of intelligent links but also the form of crisscross association superposition of human operation [4]. Derived events are inseparable from public opinion guiding subject, original events, and social environment [5]. Environment, information, or subject alienation, especially false, breaking news, and misleading information, are the main factors that cause the original public opinion to deviate from the law of evolution and generate new public opinion [6]. The deep-seated elements (i.e., group inertia cognition and judgment and collective memory), interest demand, event extension, information asymmetry, and other practical elements are the main factors leading to the emergence of a large number of derived public opinions [4]. Furthermore, the fuzziness, relevance, and irritation of the event are the direct causes of the generation of derivative public opinion, and the event participants, especially their respective interests, are the ultimate causes [7].

There are two types of models: the public opinion derivative effect model and the derivative public opinion transmission structure model. The public opinion derivative effect model is based on the logistic model. The propagation characteristics of positive and negative derivative public opinion by calculating the stable equilibrium point of the model under different information dissimilation conditions [8]. On this basis, Lian et al. [9] constructed the derivative public opinion early warning model by combining the Logistic model, exponential smoothing method, and grey prediction. Zhang et al. [10] introduced social function and individual memory into the topic-derived SEIRS public opinion transmission model. Through numerical simulation, the influence of derivative effect and related factors on the law of public opinion dissemination is revealed. In terms of the derived public opinion communication structure model, three types of derived public opinion communication structure models (i.e., direct connection, Bayesian, and radiation convergence) are discussed and the corresponding calculation methods of the derived probability are provided $[11,12]$. Gao and Wang [13] proposed five derivative public opinion propagation structures and derived probability calculation methods, such as one-way linear, composite fission, and focus convergence.

Some scholars constructed the public opinion resonance models based on Langevin's equation, in which public 
opinion event topic factors, regional factors, public opinion heat, public opinion subject attitude, and original public opinion index as moderators and validate the model through specific case simulations [14]. Li [15] further verified the model using two cases on the front and back. However, the analysis found that (1) the topic factors and regional factors in the original model were mainly the external environment factors of public opinion events, without considering the topic-related factors between public opinion events; (2) the subject attitude factor only considers the internal emotional intensity (the real emotion reflected by the self-published text). It ignores the external emotional intensity (the emotional preferences and attention of external people); (3) the corresponding regulating variable parameters are different according to the public opinion resonance phenomenon, but the original model does not consider the time series of the two resonance events; (4) the calculation of other factors as moderators is also lacking in science.

According to the review of the relevant literature above, it can be found that, although the current research on the Internet public opinion resonance has attracted the attention of scholars, the results are rare and mainly focus on the simple correlation problem of Internet public opinion in two events. However, the research on the issue of single event public opinion correlation can no longer meet the research needs of the complex, related, serialized phenomenon revealing, and rule mining of network public opinion among multiple topics related emergencies in the real world. Therefore, it is necessary to comprehensively study the topic-relevant network public opinion resonance problem with multiple specific emergencies as the object and propose new ideas and feasible solutions for the research of complex network public opinion in the current social media environment.

\section{Methodology}

Section 3.1 analyzes the influence factors of the public opinion resonance. Section 3.2 develops the Internet public opinion stochastic resonance model based on Langevin's equation.

\subsection{Influence Factors of Internet Public Opinion Resonance.} The influencing factors of Internet public opinion resonance in the public health emergencies come from many aspects, including the topic relevance of events, the field environment, the heat of original events, the emotional intensity of subjects, and the influence of evoked events.

\subsubsection{Topic-Related Factors (a). During the occurrence of} significant original public opinion events, netizens who are still in the excitement cycle of public opinion will continuously dig out the event materials of similar topics or similar subjects in the same period. Moreover, the historical material and collective memory of similar events in the past will be activated and new public opinion hotspots will be found from these materials [4]. Therefore, the higher the similarity between the original public opinion events and the topics and subjects of public opinion events in history, the greater the possibility that these "class topic," "class subject," and "class problem" events will be mined or rekindled.

3.1.2. Field Environmental Factors (b). The region and location are the central links of the network society, and the location of the event will directly affect the concerning extent of netizens [14]. Due to the different levels of economic, political, cultural, and Internet technology development in different regions, there are different degrees of attention and participation in the same issue. Furthermore, the netizens in the same region have different sensitivities to different issues of public opinion. Therefore, netizens in the same area have different influences and effects on different issues.

The calculation methods of the field environmental factors of issues, the field environmental factors of regions and the field environmental factors are as follows:

$$
\left\{\begin{array}{l}
i_{d h}=\frac{N_{d h}}{\sum_{h=1}^{n} N_{d h}}, \\
r_{h d}=\frac{N_{h d}}{\sum_{d=1}^{m} N_{h d}}, \\
b_{d h}=\frac{i_{d h}+r_{h d}}{2} .
\end{array}\right.
$$

where $i_{d h}$ is defined as the field environmental factors of the area $(d)$ about the issue $(h), r_{h d}$ is defined as the field environmental factors of the issue $(h)$ in the region $(d), b_{d h}$ is defined as the field environmental factor of the region $(d)$ and the issue (h), $N_{d h}$ is defined as the number of the public opinion events of the region $(d)$ about the issue $(h)$, and $N_{h d}$ is defined as the number of the public opinion events of the issue $(h)$ in the region $(d)$.

3.1.3. Heat of Original Events (A). Because the heat of original events is directly proportional to the attention of netizens, the heat of original events directly affects the occurrence of Internet public opinion resonance. The higher the degree of attention and discussion on this event by netizens, the longer the evolution cycle of public opinion will be. Also, it is easier for more netizens to participate in the team of digging and activating similar events, which increases the possibility of public opinion resonance.

The event heat index can objectively reflect the attention degree of events on the Internet. The larger the value of the heat index, the higher the network attention of the event. This paper mainly refers to the network propagation heat index algorithm jointly launched by the News Media Center of the School of Journalism and Communication of Tsinghua University and Sina MicroBlog to calculate the heat of original events. Firstly, we search for the data of this event under each data source through the public opinion retrieval platform. However, due to the limitation of the retrieval platform, the number of government data sources cannot be obtained. Therefore, its weights are evenly 
distributed to news, electronic newspapers, clients, WeChat public accounts, and foreign media platforms. The calculation formulas for each type of composite coefficients are as follows:

$$
\left\{\begin{array}{l}
X_{1}=0.223 * N_{\text {news }}+0.208 * N_{\text {newspapers }}+0.221 * N_{\text {client }}+0.215 * N_{\text {wechat }}+0.133 * N_{\text {foreignmedia }} \\
X_{2}=0.319 * N_{\text {weibo }}+0.355 * N_{\text {forum }}+0.326 * N_{\text {abundantguest }} \\
X_{3}=N_{\text {video }} \\
X_{4}=N_{\text {other }}
\end{array}\right.
$$

Then, each type of composite coefficient is made heat standardization so that the heat value of each type is between 0 to 1 . The normalized formula is as follows:

$$
\left\{\begin{array}{l}
Y_{i}=\frac{2}{1+\gamma_{i}^{-x_{i}}}-1, \\
\gamma_{1}=1.050 \\
\gamma_{2}=1.001 \\
\gamma_{3}=1.100 \\
\gamma_{4}=1.005
\end{array}\right.
$$

Finally, the total propagation heat index $(A)$ is synthesized by adding weights. The formula for calculating the total propagation heat index is as follows:

$$
\left\{\begin{array}{l}
A=\lambda_{1}{ }^{*} Y_{1}+\lambda_{2}{ }^{*} Y_{2}+\lambda_{3}{ }^{*} Y_{3}+\lambda_{4}{ }^{*} Y_{4}, \\
\lambda_{1}=40 \%, \\
\lambda_{2}=45 \%, \\
\lambda_{3}=5 \%, \\
\lambda_{4}=10 \% .
\end{array}\right.
$$

3.1.4. Emotional Intensity of the Participants $(f)$. From the perspective of ontology, netizens are the subject of the generation of network public opinion and the main recipient of public opinion information, whose reaction and participation to information directly affect the development trend of network public opinion. The government is the main body controlling and governing the network public opinion and plays the role of a regulator in the development of public opinion. The media is the carrier of network public opinion and promotes the fermentation of network public opinion. Opinion leaders who are the key nodes in the formation of evoked public opinion, the decoder of the source, and the encoder of the sink influence the development of public opinion events $[16,17]$. The sentiment or emotional elements of these participants are the catalysts for the generation and development of network public opinions. Under the environment of "we media," the spatial circle of public opinion is prominent. Stakeholders of all circles express mutual support, infection, and cohesion through their viewpoints to promote the unity of emotions in the whole circle and form a community of public opinion with the resonance at the same frequency. When emotional energy accumulates to a certain threshold, it promotes resonance of the emotional disclosure and collective action $[7,18,19]$.

The emotional intensity of the participants includes internal and external emotions. The calculation of the internal emotion intensity $\left(f_{\text {in }}\right)$ is mainly performed by the model API interface, which belongs to the Boson NLP sentiment analysis and is trained by the MicroBlog corpus. It can calculate the emotion value of every MicroBlog text for each participant. The external emotion intensity $\left(f_{\text {out }}\right)$ is calculated for each MicroBlog text by WeberFechner Law and the first law of emotional intensity. Because emotion is a personal subjective reflection, it is the physiological process that human brain motive and feel the value characteristics of things. In the value characteristics of things, "value rate" and "value rate difference" are the most important, which fundamentally determine the development direction of things and the human's attitude toward it. And, the relationship between emotional intensity and relative value intensity (the contrast in value rate of things) follows WeberFechner Law that emotional intensity is proportional to the logarithm of the difference in the rate of value of things. The calculation process is shown as follows:

$$
\left\{\begin{array}{l}
f_{\text {out }}=K_{m} \log (1+\Delta \psi), \\
\Delta \psi=\frac{2 N_{z f} N_{d z}}{N_{z f}+N_{d z}}
\end{array}\right.
$$

where $f_{\text {out }}$ is the external emotion intensity; $K_{m}$ is the emotional intensity coefficient; $\Delta \psi$ is the contrast of value rate; $N_{z f}$ is the number of Microblog forwarding; and $N_{d z}$ is the number of Microblog praise.

Then, the emotional intensity of each MicroBlog is obtained by weighted summation. After that, the average emotion intensity of each subject's MicroBlog is extracted as the emotional intensity of its subject. Then, the emotional intensity of the participants $(f)$ is obtained by weighting and summing the emotional intensity of each participant. The weight of the emotional intensity of each subject for the influence on the resonance of public sentiment refers to the paper of $\mathrm{Li}$ [20] who calculated weights by inviting expert scores and AHP, which is " $\beta_{\text {gov }}=0.40, \beta_{\text {media }}=0.25$, $\beta_{\text {leader }}=0.20, \beta_{\text {netizens }}=0.15$." The calculation method of the 


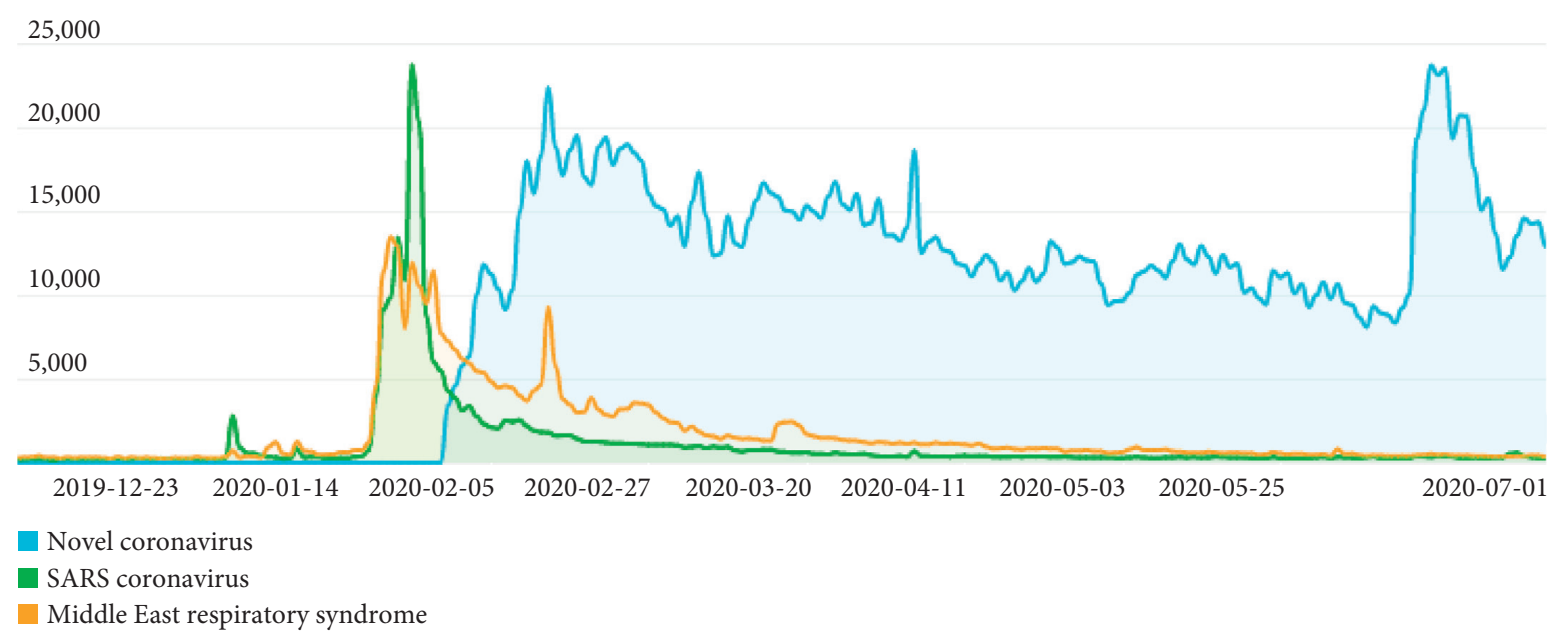

Figure 1: COVID-19 public opinion resonance event clusters.

emotional intensity of each subject and the event are as follows:

$$
\left\{\begin{array}{l}
f_{z t}=\frac{f_{\mathrm{in}_{z t}}+f_{\mathrm{out}_{z t}},}{2} \\
f=\sum_{z t=1}^{m} \beta_{z t} * f_{z t},
\end{array}\right.
$$

where $f_{z t}$ is the emotional intensity of each subject and $\beta_{z t}$ is the weight of the emotional intensity of each subject.

3.1.5. Influence of Evoked Events (D). The first cause effect in psychology is "preconceived." It is believed that the information first entered or received will form the first impression memory schema in the human brain, and subsequent judgments will be integrated into this memory schema and have the traces of previous information attributes [21]. Moreover, some factors, such as the collective memory of netizens, can contribute to the generation of related events. The greater the influence of evoked events, the deeper memory in the netizens, and when similar events occur, it will easily emerge in the minds of the netizens and activate to revive.

3.2. The Model. In this section, we propose the Internet public opinion stochastic resonance model based on Langevin's equation.

Stochastic resonance (SR) was first discovered and proposed by Benzi (1981). Some scholars applied SR model in social finance, group behavior, and other social science disciplines. For example, Liu [22] proposed theory of Financial Brownian Particle (FBP) to simulate limit order market is developed. Schenck et al. [23] proposed a generalized Langevin process with asymmetric double-well potential function, to model the information flow and price discovery process. In this paper, the Internet public opinion stochastic resonance model of the bistable system is established based on the aforementioned influence factors of the public opinion resonance. In the model, the topic-related events are considered. In addition, the bistable system subjecting to noise $\Gamma(t)$ and external periodic driving forces $\left(A \cos \omega_{0} t\right)$ is described by the Langevin equation (LE), as shown in

$$
\dot{x}=a x-b x^{3}+A \cos \omega_{0} t+\Gamma(t) .
$$

In equation (7), $a$ is the topic-related factor between events; $b$ is the field environment factor, and $\left(f(x)=a x-b x^{3}\right)$ is the nonlinear external force field with a double-well. $a$ and $b$ are the shape parameters of the potential well, which have a great influence on the model characteristics and directly affect the effect of stochastic resonance; and $A$ is set as the heat of the original events, which is affected by the emotional intensity of the participants in public opinion events $f\left(\omega_{0}=2 \pi f\right)$. The participants mainly include government departments, media, opinion leaders, and netizens, whose emotional intensity directly affects the trend of events. $\Gamma(t)=\sqrt{2 D} \omega(t)$ is the added Gaussian white noise, which noise intensity $(D)$ corresponds to the influence of evoked events. $X$ represents the displacement of moving particles in the bistable system, and $t$ represents time.

The fourth-order RungeKutta algorithm is a high-precision single-step method and can reach the fourth-order accuracy. In addition, only the first derivative is needed to be known, and no other high-order derivatives need be defined or calculated. It also has the advantages of good numerical stability, self-starting, and easy programming. So, we use the fourth-order RungeKutta algorithm to solve equation (7).

\section{Case Study}

4.1. Event Review of the Internet Public Opinion. In this study, this article follows the screening criteria of public health emergencies with certain influence and similar topic or subjects that can be retrieved in the event database of the 
"knowledge of micromatters" platform and the "Baidu Index." In order to avoid the contingency of experimental results due to the use of only two events, we finally selected three cases of vaccine public health emergencies in China as an example. Three cases of public health emergencies in China are taken as examples, the Shandong illegal vaccine event was selected as an evoked event; the Chongqing switched vaccine event was selected as the original event I; the Changsheng faked vaccine event was selected as the original event II. The phenomenon of Internet public opinion resonance caused by these three events will be studied.

4.1.1. Shandong Illegal Vaccine Event. On March 18, 2016, a woman and her daughter in Jinan, Shandong province, were suspected of illegally selling vaccines. Once exposed, it attracted the attention and discussion of netizens all over the country. The number of reposts and searches continued to increase, and the event began to ferment. On March 22, Premier Li Keqiang made important instructions on a series of cases of illegally operating vaccines and demanded a thorough investigation of the flow and use of "problem vaccines," at which time public opinion entered a climax. On April 13, Premier Li Keqiang presided over a meeting to hear a report on the investigation and handling of the case. According to the investigation results, he punished that relevant responsible person and those relevant responsible people following the law. At this point, the event entered a period of receding. On July 15, 2018, following the Changsheng faked vaccine event, the Shandong illegal vaccine event once again aroused the attention and discussion of netizens. With relevant enterprises ordered to halt production and file a case for investigation, the State Food and Drug Administration identified the flow of substandard vaccines. On July 22, Internet public opinion reached a peak.

4.1.2. Chongqing Switched Vaccine Event. On May 13, 2016, when the Shandong illegal vaccine event just happened less than two months ago, it caused concern on the Internet that some parents in Chongqing suspected that the community clinics have "switched" vaccines. Surrounding residents gathered at the service center that night to demand to know the fact. After that, the Chongqing Municipal Party Committee and other departments immediately launched an investigation overnight. On May 14, the joint investigation team announced the latest progress of the investigation through the official MicroBlog. At this time, the attention and searching value of the event reached a peak. On May 16th, the joint investigation team announced that four involved had been detained for profit-seeking. Since then, the attention and discussion of this event have entered a recession. On July 15, 2018, with the Changsheng faked vaccine event, this event also attracted the attention of netizens, but in comparison, it was relatively smaller and did not cause large fluctuations.

4.1.3. Changsheng Faked Vaccine Event. On July 15th, 2018, Changchun Changsheng Biochemical Co., Ltd. was investigated for producing rabies vaccine illegally. Once the event was disclosed, it quickly aroused online discussion and continued to ferment. On July 16, Changsheng Biological Company apologized and recalled all rabies vaccines, which was within the validity period. Meanwhile, Prime Minister Li Keqiang has issued instructions on the vaccine event and demanded a thorough investigation. Internet searches for the event have soared. On July 23, with the CCTV news broadcast about this case, the Disease Control Departments in 20 provinces and cities collectively stopped using the Changchun Changsheng rabies vaccines. The Internet's attention and search for the event were into the climax. From July 24 to 26, with the arrest of the chairman of this company and the other 14 people involved in the case, the fact that the company has illegally produced rabies vaccines has been found out, and the event has temporarily entered a recession.

\subsection{Parameter Calculation of Internet Public Opinion Resonance Model}

4.2.1. Topic-Related Factors (a). In order to calculate the topic-related degree between the original events and the evoked event, "Shandong AND Vaccine," "Chongqing AND Vaccine," and "Changsheng AND Vaccine" were used as keywords to search through MicroBlog. The Shandong illegal vaccine event (March 18, 2016, from May 12, 2016, 2933 articles; March 18, 2016, from July 14, 2018, 3799 articles), Chongqing switched vaccine event (May13, 2016, from June 30, 2016, 604 articles) and Changsheng faked vaccine event (July 15, 2018, from July 30, 2018, 6581 articles) were taken to use as sample data. Among them, the Shandong illegal vaccine event was divided into two sample data sets, which are from the time when the Shandong illegal vaccine event started to one day before to the time when the two original events started. Because the development process of each public opinion event will generate multiple discussion topics, the two events which have any two similar discussion topics and subjects will most likely be mined suggestively. Therefore, this study calculates the similarity between topics of the two public opinion events and extracts the group with the highest similarity as the topic-related factors $(a)$ of the public opinion resonance model.

Firstly, preprocessing operations such as separating words, adding a custom vocabulary, and removing stop words are performed on the MicroBlog sample data of each public opinion event. Then, the LDA (Latent Dirichlet Allocation) model is used to extract the topic of each public opinion event. To ensure the independence of the topics, the number of topics for the event should be the number of topics when the event topics do not cross. Next, the top 10 topic tokens of each topic of events are extracted as corpora and transformed into a vector (bow), shown in Table 1.

Further, the vector (bow) is transformed into the TF-IDF topic model vector, and cosine similarity is used to calculate the similarity between one event topic vector and another. The maximum value of the similarity between the two topics is selected as the topic relevance of these two events. The results are shown in Table 2. By calculation, the topic-related degree between original event I (Chongqing switched 
TABLE 1: The top 10 topic tokens of each topic events.

\begin{tabular}{|c|c|c|}
\hline Event & Number & Topic token (top 10) \\
\hline \multirow{4}{*}{$\begin{array}{l}\text { Shandong illegal vaccine event I } \\
(2016.03 .18 \sim 2016.05 .12)\end{array}$} & A1 & $\begin{array}{c}\text { ["Vaccine," "Shandong," "Illegal Operation," "Inflow," "Vaccination," "Cold } \\
\text { Chain," "Cold Storage," "Children," "Type II," "Jinan"] }\end{array}$ \\
\hline & $\mathrm{A} 2$ & $\begin{array}{l}\text { ["Vaccine," "Shandong," "Illegal Operation," "Handle," "Food and Drug," } \\
\text { "Suspects," "Problematic vaccine," "Jinan," "Shandong Province," "Flow"] }\end{array}$ \\
\hline & A3 & $\begin{array}{l}\text { ["Vaccine," "Shandong," "Enterprise," "Drug," "FDA," "Illegal Operation," } \\
\text { "Biological product," "Shandong province," "Operate," "Sell"] }\end{array}$ \\
\hline & A4 & $\begin{array}{l}\text { ["Vaccine," "Shandong," "Illegal Operation," "Bilk," "Compensation," "Sichuan," } \\
\text { "Problematic Vaccine," "Problem," "Inject," "Fabricate"] }\end{array}$ \\
\hline \multirow{3}{*}{$\begin{array}{l}\text { Shandong illegal vaccine event II } \\
\text { (2016.03.18 2018.07.14) }\end{array}$} & B1 & $\begin{array}{l}\text { ["Vaccine," "Shandong," "Illegal Operation," "Illegal," "Enterprise," "FDA," } \\
\text { "Drug," "Shandong province," "Food and Drug," "Operate"] }\end{array}$ \\
\hline & B2 & $\begin{array}{c}\text { ["Vaccine," "Shandong," "Illegal Operation," "Illegal," "Trickster," "Problematic } \\
\text { Vaccine," "Bilk," "Vaccination," "Inflow," "Cold Chain"] }\end{array}$ \\
\hline & B3 & $\begin{array}{l}\text { ["Vaccine," "Shandong," "Illegal Operation," "Illegal," "Pang Hongwei," "Crime," } \\
\text { "Approve Arrest," "Sentence," "Supervise," "Arrest"] }\end{array}$ \\
\hline \multirow{3}{*}{$\begin{array}{l}\text { Chongqing switched vaccine event } \\
(2016.05 .13 \sim 2016.06 .30)\end{array}$} & $\mathrm{C} 1$ & $\begin{array}{l}\text { ["Vaccine," "Injection," "Package," "Chongqing," "Nurse," "Vaccination," } \\
\text { "Original," "Targeted," "Doubt," "Health Service Center"] }\end{array}$ \\
\hline & $\mathrm{C} 2$ & $\begin{array}{l}\text { ["Vaccine," "Chongqing," "Package," "Investigation Team," "Suspect," "Hospital," } \\
\text { "Chongqing," "Investigation," "Health Service," "Doubt"] }\end{array}$ \\
\hline & $\mathrm{C} 3$ & $\begin{array}{l}\text { ["Vaccine," "Chongqing," "Package," "Nurse," "Contaminated Syringe," "Crime," } \\
\text { "Medical Service," "Doctors," "Regular," "Problem"] }\end{array}$ \\
\hline \multirow{5}{*}{$\begin{array}{l}\text { Changsheng faked vaccine event } \\
\text { (2018.07.15 2018.07.30) }\end{array}$} & D1 & $\begin{array}{l}\text { "Problem," "Disqualified," "Fraudulent Production Records," "company," } \\
\text { "problematic Vaccine"] }\end{array}$ \\
\hline & D2 & $\begin{array}{c}\text { ["Vaccine," "Rabies," “Company," "Freeze-dried," “Crime," "Changchun } \\
\text { Changsheng Biological Technology Limited Company," "FDA," "Enterprise," } \\
\text { "Fake," "Records"] }\end{array}$ \\
\hline & D3 & $\begin{array}{c}\text { ["Vaccine," "Company," "Rabies," "Freeze-dried," "Fake," "Changchun } \\
\text { Changsheng Biological Technology Limited Company," "Jilin Province," "Food } \\
\text { and Drug," "Sell," "Biology"] }\end{array}$ \\
\hline & $\mathrm{D} 4$ & $\begin{array}{c}\text { ["Vaccine," "Rabies," "Violation," "Company," "Security," "Keqiang Li," "Fake," } \\
\text { "Fraudulent Production Records," "Enterprise," "Medicate"] }\end{array}$ \\
\hline & D5 & $\begin{array}{l}\text { ["Vaccine," "Rabies," "Violation," "Enterprise," "company," "original," } \\
\text { "Investigation Team," "Product," "Investigate Clearly," "Preparation"] }\end{array}$ \\
\hline
\end{tabular}

vaccine event) and evoked event (Shandong illegal vaccine event I) is 0.40 , and the topic-related degree between the original event II (Changsheng faked vaccine event) and evoked event (Shandong illegal vaccine event II) is 0.58 . Therefore, the topic correlation degree between the Chongqing switched vaccine event and the Shandong illegal vaccine event is less than that between the Changsheng faked vaccine event and the Shandong illegal vaccine event.

4.2.2. Field Environmental Factors (b). Relying on the hot events recorded in the public opinion events data base of the official website of "knowledge of micromatters," which can reach a high transmission volume in a short time and maintain a certain amount of transmission for a long time, this study counts all public opinion events recorded in the event database from evoked events to original events I and original events II, that is, data from March 2016, to July 2018. Still, the data do not include Hong Kong, Macau, Taiwan, and international events and those events for which the source of the incident is not known through other channels. First of all, according to the location of the incident (the seven administrative regions of Northeast, North China, East China, Central China, South China, Northwest, and Southwest) and combined with Liao et al.'s concept of dividing five types of public opinion events and the event type tag of the public opinion events database of the official website, the event was divided into five event types (emergencies, social livelihood, sports and entertainment, enterprise finance, and current affairs politics) and the public opinion information in this period is classified. Then, the study calculated the proportion of different topics in the same area (the field environmental factors of issues) and the proportion of different areas in the same issue (the field environmental factors of regions). The results are shown in Table 3. The contents in brackets are the value of the field environmental factors of issues and the field environmental factors of regions. Finally, the field environmental factors $(b)$ of each original event and evoked event are calculated by weighted summation.

Original event I and original event II are both the social livelihood events. The original event I occurred in Chongqing (southwest) in May 2016, where the field environmental factors of issue and region are (0.60/0.05), while the original event II occurred in Changchun (northeast) in July 2018, where the field environmental factors of topic and region are (0.54/0.04). After weighted summation, the values of field environmental factors $(b)$ for original event I and original event II are, respectively, 0.33 and 0.29 . 
TABLE 2: Topic correlation degree between original events and evoked event.

\begin{tabular}{|c|c|c|}
\hline Topic correlation degree & Number & Similarity \\
\hline \multirow{11}{*}{0.40} & $\mathrm{C} 1-\mathrm{A} 1$ & 0.40 \\
\hline & $\mathrm{C} 1-\mathrm{A} 2$ & 0.00 \\
\hline & $\mathrm{C} 1-\mathrm{A} 3$ & 0.00 \\
\hline & $\mathrm{C} 1-\mathrm{A} 4$ & 0.00 \\
\hline & $\mathrm{C} 2-\mathrm{A} 1$ & 0.00 \\
\hline & $\mathrm{C} 2-\mathrm{A} 2$ & 0.00 \\
\hline & $\mathrm{C} 2-\mathrm{A} 3$ & 0.00 \\
\hline & $\mathrm{C} 2-\mathrm{A} 4$ & 0.00 \\
\hline & C3-A1 & 0.40 \\
\hline & $\mathrm{C} 3-\mathrm{A} 2$ & 0.00 \\
\hline & $\mathrm{C} 3-\mathrm{A} 3$ & 0.00 \\
\hline \multirow{16}{*}{0.58} & C3-A4 & 0.00 \\
\hline & D1-B1 & 0.58 \\
\hline & D2-B1 & 0.47 \\
\hline & D3-B1 & 0.41 \\
\hline & D4-B1 & 0.41 \\
\hline & D5-B1 & 0.41 \\
\hline & D1-B2 & 0.00 \\
\hline & D2-B2 & 0.24 \\
\hline & D3-B2 & 0.00 \\
\hline & D4-B2 & 0.00 \\
\hline & D5-B2 & 0.00 \\
\hline & D1-B3 & 0.00 \\
\hline & D2-B3 & 0.00 \\
\hline & D3-B3 & 0.00 \\
\hline & D4-B3 & 0.00 \\
\hline & D5-B3 & 0.00 \\
\hline
\end{tabular}

4.2.3. Heat of Original Events (A). Using the specialized public opinion retrieval platform of "Digudata" and "MeiyaPICO," "Chongqing AND Vaccine" and "Changsheng AND Vaccine" were used as keywords, and the time limit was May 13, 2016, to June 30, 2016, and July 15, 2018, to July 30, 2018; we retrieved and counted the number of data under each data source of the two original events within their respective time limits. The results are shown in Table 4. Finally, the event as mentioned above, heat index algorithm was used to calculate the heat value $(A)$ of the original event I and original event II, which are 0.26 and 0.92 .

4.2.4. Emotional Intensity of the Participants (f). With reference to the above calculation method of emotional intensity, the internal and external emotional intensity of the public opinion information of the original event is calculated, respectively, for the four types of the MicroBlog participants: government, media, opinion leaders, and Internet users. The classification of the main categories is mainly based on Sina MicroBlog V certification system. This paper classifies the relevant government departments among "Blue V" users as government subject, "Gold V" users are self-media users. The "Gold V" self-media users are combined with the traditional media in "Blue V," which are both classified as media subject. Due to a large number of fans and readings of the "Yellow V" users, it is classified as the opinion leader subject. And, the remaining "MicroBlog experts," "MicroBlog members" and "ordinary users" are uniformly classified as Internet users. In the end, the internal and external emotional intensities of each participant and the overall emotional intensity of the participants in the original event I and original event II are shown in Table 5:

4.2.5. Influence of Evoked Events (D). According to the calculating method of event influence index on the platform of "knowledge of micromatters" the Event Influence Index (EII) is a reliable indicator for describing the propagation effect of a single event. Firstly, calculate the channel influence of each data release in each social media and network media platform, sum up and normalize the data to the $[0,100]$ range, and get the influence index of its subplatform events; then, according to the comprehensive evaluation of the communication effect of the event on the three platforms of online media, MicroBlog, and Wechat, the influence index of the event is finally obtained by weighted calculation and normalization. Because of the evoked events in this paper for original event I and original event II are the same Shandong illegal vaccine events, the event influence index is extracted from the event database and normalized to $[0,1]$ as the influence of evoked events $(D)$. Among them, the MicroBlog influence index of the Shandong illegal vaccine event was 81.5 , the WeChat influence index was 86.8, the Internet media influence index was 78.4, and the comprehensive evaluation of propagation effects in the three platforms is weighted to calculate the normalized influence index of the entire event to be 81.4 , that is, the value of evoked event influence $(D)$ is 0.81 . 
TABLe 3: Numbers and proportions of different topics by each area.

\begin{tabular}{|c|c|c|c|c|c|c|c|}
\hline Time & Area & Emergencies & $\begin{array}{c}\text { Social } \\
\text { livelihood }\end{array}$ & $\begin{array}{c}\text { Sports and } \\
\text { entertainment }\end{array}$ & $\begin{array}{l}\text { Enterprise } \\
\text { finance }\end{array}$ & $\begin{array}{l}\text { Current affairs } \\
\text { (politics) }\end{array}$ & Sum \\
\hline \multirow{7}{*}{ 2016.03-2016.05 } & Northeast & $1(0.17 / 0.05)$ & $3(0.50 / 0.03)$ & $1(0.17 / 0.02)$ & $0(0.00 / 0.00)$ & $1(0.17 / 0.04)$ & 6 \\
\hline & North China & $5(0.06 / 0.26)$ & $25(0.297 / 0.23)$ & $29(0.33 / 0.57)$ & $8(0.09 / 0.53)$ & $20(0.23 / 0.87)$ & 87 \\
\hline & East China & $8(0.12 / 0.42)$ & $44(0.64 / 0.40)$ & $11(0.16 / 0.22)$ & $6(0.09 / 0.40)$ & $0(0.00 / 0.00)$ & 69 \\
\hline & $\begin{array}{l}\text { Central } \\
\text { China }\end{array}$ & $1(0.06 / 0.05)$ & $12(0.71 / 0.11)$ & $3(0.18 / 0.06)$ & $0(0.00 / 0.00)$ & $1(0.06 / 0.04)$ & 17 \\
\hline & South China & $1(0.05 / 0.05)$ & $14(0.70 / 0.13)$ & $3(0.15 / 0.06)$ & $1(0.05 / 0.07)$ & $1(0.05 / 0.04)$ & 20 \\
\hline & Northwest & $1(0.10 / 0.05)$ & $7(0.70 / 0.06)$ & $2(0.20 / 0.04)$ & $0(0.00 / 0.00)$ & $0(0.00 / 0.00)$ & 10 \\
\hline & Southwest & $2(0.20 / 0.11)$ & $6(0.60 / 0.05)$ & $20.20 / 0.04)$ & $0(0.00 / 0.00)$ & $0(0.00 / 0.00)$ & 10 \\
\hline \multirow[t]{4}{*}{ Sum } & & 19 & 111 & 51 & 15 & 23 & 219 \\
\hline & Northeast & $9(0.13 / 0.09)$ & $36(0.54 / 0.04)$ & $7(0.10 / 0.02)$ & $12(0.18 / 0.03)$ & $3(0.04 / 0.02)$ & 67 \\
\hline & North China & $18(0.02 / 0.17)$ & $277(0.32 / 0.30)$ & $235(0.28 / .64)$ & $181(0.21 / 0.40)$ & $142(0.17 / 0.78)$ & 853 \\
\hline & East China & $32(0.06 / 0.30)$ & $294(0.52 / 0.32)$ & $72(0.13 / 020)$ & $150(0.26 / 0.33)$ & $21(0.04 / 0.11)$ & 569 \\
\hline \multirow[t]{4}{*}{ 2016.03-2018.07 } & $\begin{array}{l}\text { Central } \\
\text { China }\end{array}$ & $8(0.07 / 0.08)$ & $80(0.72 / 0.09)$ & $17(0.15 / 0.05)$ & $5(0.05 / 0.01)$ & $1(0.01 / 0.01)$ & 111 \\
\hline & South China & $11(0.04 / 0.10)$ & $121(0.47 / 0.13)$ & $23(0.09 / 0.06)$ & $88(0.35 / 0.20)$ & $12(0.05 / 0.07)$ & 255 \\
\hline & Northwest & $8(0.13 / 0.08)$ & $46(0.74 / 0.05)$ & $4(0.06 / 0.01)$ & $2(0.03 / 0.00)$ & $2(0.03 / 0.01)$ & 62 \\
\hline & Southwest & $19(0.17 / 0.18)$ & $70(0.63 / 0.08)$ & $8(0.07 / 0.02)$ & $12(0.11 / 0.03)$ & $2(0.02 / 0.01)$ & 111 \\
\hline Sum & & 105 & 924 & 366 & 450 & 183 & 2028 \\
\hline
\end{tabular}

Table 4: Number of retrieved data for each data source of the original events.

\begin{tabular}{lccccccccccc}
\hline & News & Newspapers & Client & WeChat & Foreign media & MicroBlog & Forum & Blog & Video & Others \\
\hline Original event I & 48 & 1 & 0 & 18 & 0 & 776 & 3 & 0 & 0 & 4 \\
Original event II & 9415 & 941 & 4627 & 8182 & 291 & 579834 & 4875 & 134 & 3 & 318 \\
\hline
\end{tabular}

TABle 5: The emotional intensity of participants for each original event.

\begin{tabular}{|c|c|c|c|c|c|}
\hline & $f_{\text {gov }}\left(f_{\text {in }} / f_{\text {out }}\right)$ & $f_{\text {media }}\left(f_{\text {in }} / f_{\text {out }}\right)$ & $f_{\text {leader }}\left(f_{\text {in }} / f_{\text {out }}\right)$ & $f_{\text {netizens }}\left(f_{\text {in }} / f_{\text {out }}\right)$ & $f$ \\
\hline Original event I & $0.32(0.52 / 0.13)$ & $0.52(0.58 / 0.46)$ & $0.28(0.45 / 0.10)$ & $0.19(0.37 / 0.02)$ & 0.34 \\
\hline Original event II & $0.46(0.56 / 0.37)$ & $0.59(0.59 / 0.60)$ & $0.43(0.64 / 0.22)$ & $0.32(0.59 / 0.04)$ & 0.47 \\
\hline
\end{tabular}

4.3. Simulation of Internet Public Opinion Resonance. The parameter values of the above models are substituted into the topic-related event public opinion resonance model, that is, Chongqing switched vaccine event (original event I) and Shandong illegal vaccine event (evoked event) $(a=0.40, b=0.33, A=0.26, f=0.34, D=0.81) \quad$ and Changsheng faked vaccine event (original event II) and Shandong illegal vaccine event (evoked event) $(a=0.58, b=0.29, A=0.92, f=0.47, D=0.81)$ The fourth-order RungeKutta algorithm is implemented in MATLAB. Finally, we get the chart that can reflect the public opinion resonance effect in pair-theme related events. The results are shown in Figures 2 and 3.

It can be seen from Figure 2 that there is no visible resonance peak in the public opinion resonance spectrum of Chongqing switched vaccine event (original event I) and Shandong illegal vaccine event (evoked event), and the spectrum amplitude is smaller, which indicates that there is an under-resonance between the two public health emergencies. The Chongqing switched vaccine event did not trigger a renewed discussion among netizens on the subject of Shandong illegal vaccine even.
It can be seen from Figure 3 that there is a clear peak in the public opinion resonance spectrum for Changsheng faked vaccine event (original event II) and Shandong illegal vaccine event (evoked event). When the frequency is $0.007813 \mathrm{f} / \mathrm{Hz}$, the spectrum amplitude reaches a maximum of 641.5, indicating that between these two public health emergencies emerge the phenomenon of stochastic resonance. In other words, the Changsheng faked vaccine even raised again heated discussion among netizens about the related topic of Shandong illegal vaccine event, and the resonance and superposition of the two events will make netizens' discussion of the topic enter a high level again. The level of attention and influence goes far beyond the primary single public health emergency.

\section{Validation Experiment and Discussion}

5.1. Model Effect Validation. In order to verify the effectiveness of the public opinion resonance model in the above three public health emergencies, this study obtained the Baidu Index Trend of three public health emergencies in the period from January 1, 2016, to December 31, 2018. The data 


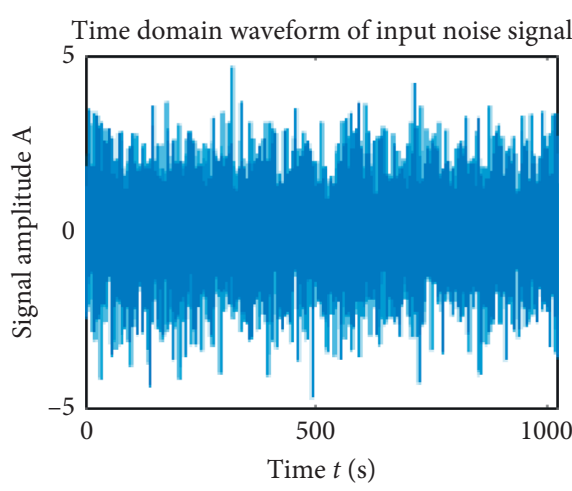

(a)

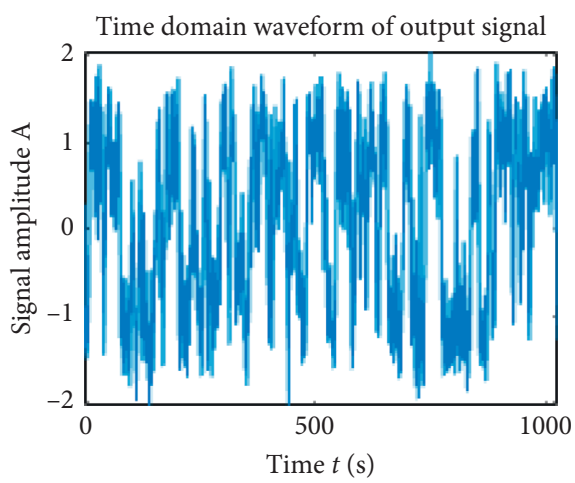

(c)

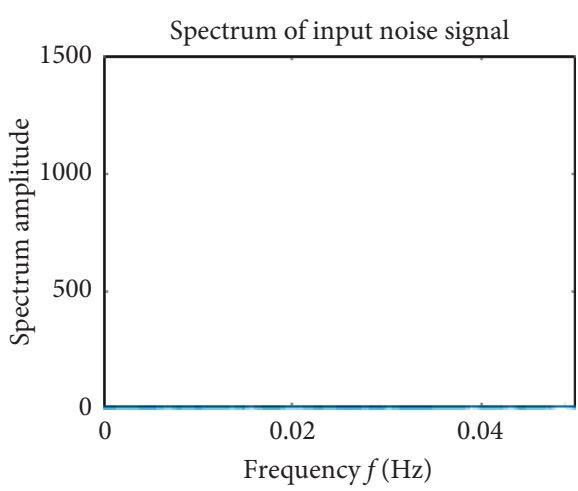

(b)

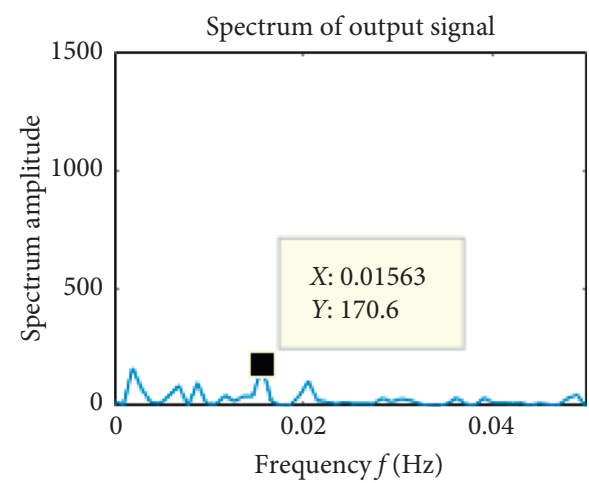

(d)

FIGURE 2: Chongqing switched vaccine event-Shandong illegal vaccine event resonance diagram.

were collected through Baidu Index platform with the keywords of "Shandong vaccine event," "Chongqing vaccine event," and "Changsheng vaccine event," respectively. The results are shown in Figure 4, which is compared with the above simulation results.

By comparing the simulation results of the Langevin model considering the topical relevance of public health emergencies (Figures 2 and 3) with the Baidu public opinion index trend chart of the two groups of events (Figure 4), it can be found that the resonance effect between Chongqing transfer vaccine event (original event I) and Shandong illegal vaccine event (evoked event) is very weak, while the resonance effect between the Changsheng fake vaccine event (original event II) and Shandong illegal vaccine event (evoked event) is very significant. The reason is that the Shandong illegal vaccine incident did not completely subside at the time of Chongqing transfer vaccine incident. At the same time, Chongqing municipal government's rapid and accurate response to the incident has little social impact, so there is no obvious resonance between the two. On the contrary, the Changsheng fake vaccine event and the Shandong illegal vaccine event have caused great social impact. Both events received high attention from the national government, especially from July 22,2018 , to July 23,2018 . With the identification of the Shandong illegal vaccine event, the "longterm memory" of Internet users was aroused, which caused a strong resonance between the public opinion of the two events.

By comparing the simulation results with the Baidu index trend chart, we find that the effect of the public opinion resonance model considering topical relevance has a high degree of agreement with the real event development trend of the Baidu index. In addition, by comparing the results of the two groups of events, it can be found that the shorter the time interval between the emergency events is, the less conducive it is to stimulate the resonance of public opinion between the original event and the evoked event.

5.2. Validation of Model Sensitivity and Significance. In order to verify the sensitivity and significance of the public health emergency Internet public opinion resonance model considering the topic relevance degree compared with the original public opinion resonance model proposed by Liao and $\mathrm{Li}$, the simulation results of the two models were compared by using the Internet public opinion data of these three public health emergencies. The original model and its parameter definition and solution are as follows:

$$
\dot{x}=i x-r x^{3}+H \cos \left[\left(g \cdot p_{1}+h \cdot p_{2}+k \cdot p_{3}+z \cdot p_{4}\right) t\right]+s,
$$

where $I$ and $r$ are the factors of the issue and the regions, respectively. The values are the proportion of the number of public opinion events in different issues in the same region and different regions of the same issue. $(g, h, k$, and $z)$ are the weights of law enforcement agencies, online media, opinion leaders, and attitude of other netizens $(g+h+k+z=1), p_{i}$ is the attitude value of each subjeFct, that is, the sentiment value of the public opinion event content issued by each subject. $H$ and $s$ are the index of the 


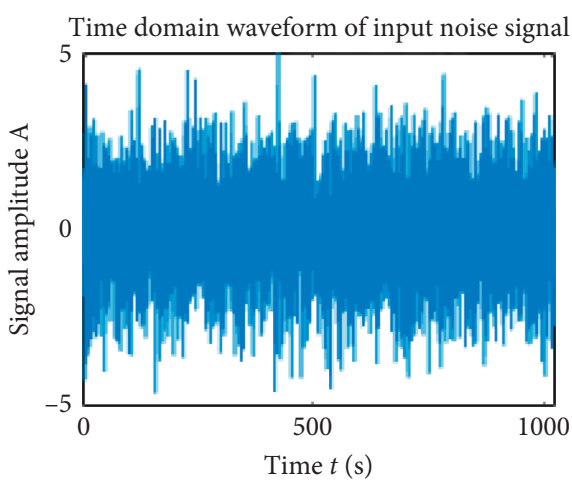

(a)

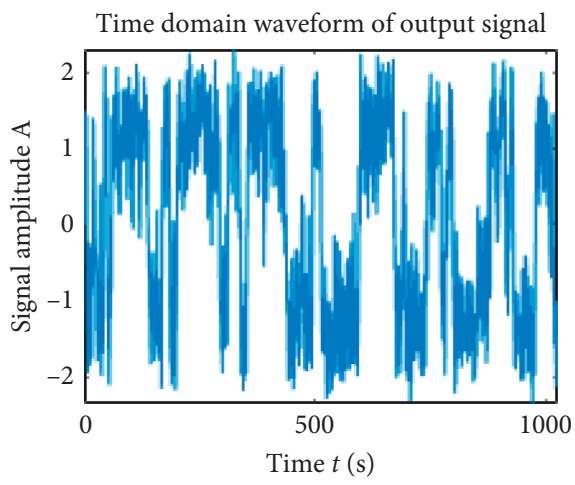

(c)

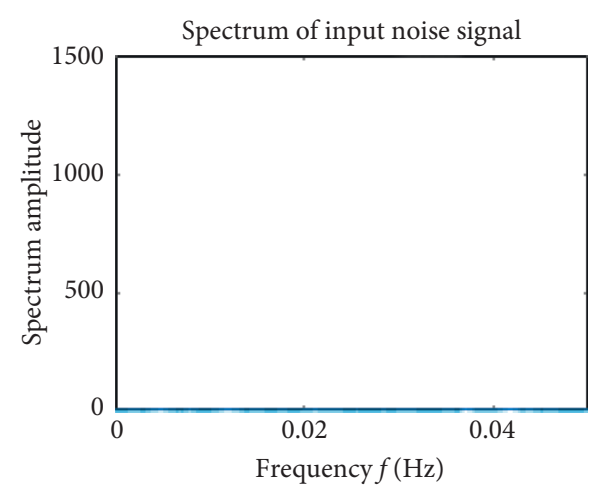

(b)

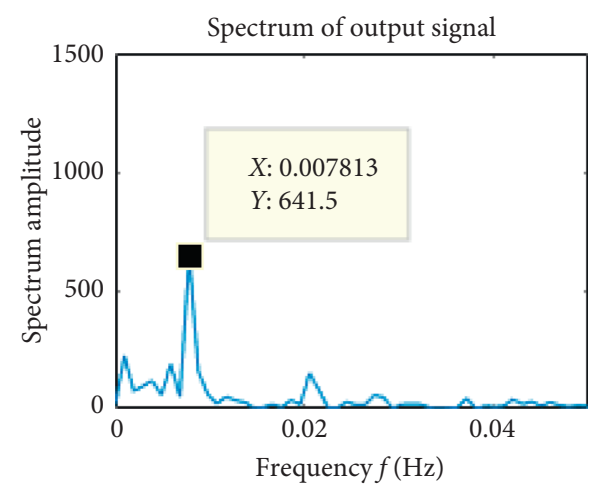

(d)

FIGURE 3: Changsheng faked vaccine event-Shandong illegal vaccine even resonance diagram.

heat of evoked events and the original event public opinion. The public opinion index evaluates the intensity of public opinion events from the number, energy, and quality of public opinion.

In this study, the values of $H$ and $s$ correspond to the values of $A$ and $D$ of each event without regard to the timing of events. At the same time, the issue factors $I$ and regional factors $r$ in the original model correspond to the values of the issue field environmental factors and the regional field environmental factors in the field environmental factors $b$ in the research model, respectively. The value of the subject attitude pin the original model is consistent with the value of internal emotion intensity $\left(f_{\text {in }}\right)$ in the emotional intensity of the participants $(f)$ in this research model. Therefore, after conversion and calculation, the parameter values corresponding to the original models of the two sets of case events are as follows: (1) Chongqing packaged vaccine incident-Shandong illegal vaccine event $(i=0.60, r=0.05, H=0.26, p=0.50, s=0.81$ ).

Changsheng faked vaccine event-Shandong illegal vaccine event $(i=0.54, \mathrm{r}=0.04, H=0.92, p=0.59, s=0.81)$. The two groups of events are separately simulated and the results are shown in Figure 5.

It can be seen that in the simulation results of the original model, two groups of events all show the phenomenon of over resonance, that is, in the public opinion resonance spectrum, the frequency spectrum amplitude is relatively high in the initial state, then plummets and approaches to zero. The phenomenon of public resonance between events is difficult to describe clearly. Comparatively, from the perspective of simulation results, the public health emergency Internet public opinion resonance model considering topical relevance is more sensitive and significant than the original public opinion resonance model.

5.3. Impact of Topic-Related Factors on Internet Public Opinion Resonance. Compared with the original model, the most important improvement of this study is that it considers the topical relevance between public opinion events to make further explorations in the law of the influence of the topicrelated factors on Internet public opinion resonance. While maintaining the values of the other factors of the two groups of events unchanged, we only adjusted the value of the topical relevance factor in the range $[0,1]$ for comparative simulation analysis, with a step size of 0.20 . The initial parameters of the two groups of events are as follows: (1) Chongqing packaged vaccine incident-Shandong illegal vaccine event ( $a=0.00, b=0.33, A=0.26, f=0.34, D=0.81$ ). (2) Changsheng faked vaccine event-Shandong illegal vaccine event $(a=0.00, b=0.29, A=0.92, f=0.47, D=0.81)$. The results are shown in Figure 6.

It can be seen from Figure 6 that with the value of the topic-related factor $(a)$ continues to increase, the amplitudes of the resonance spectrum of the two groups of events also become increasingly prominent. It shows that the topical relevance degree between two groups of public health 


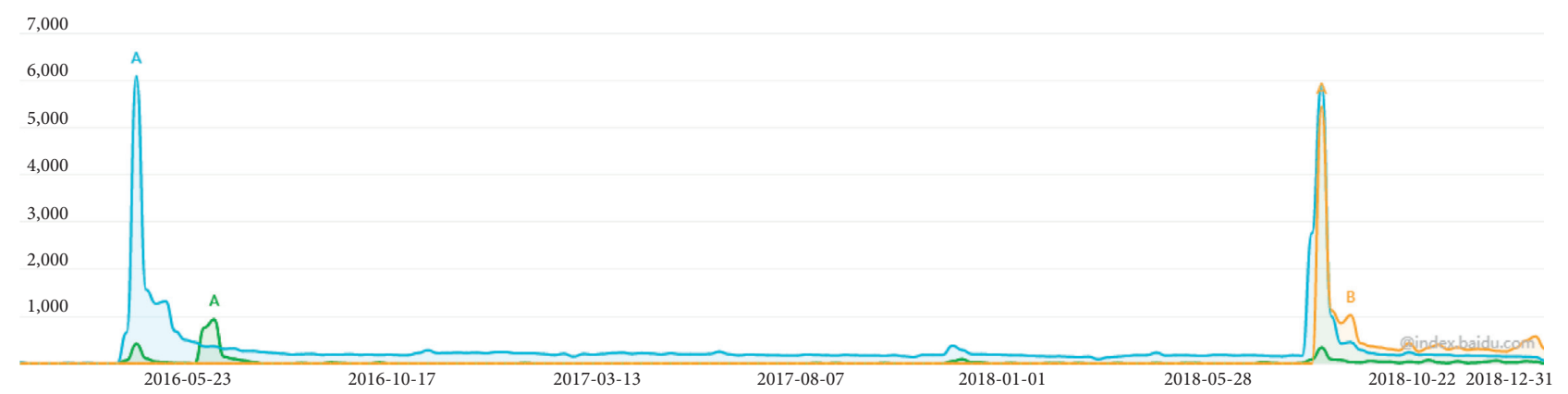

Novel coronavirus

SARS coronavirus

Middle east respiratory syndrome

Figure 4: Baidu index trend comparison chart of public opinion events.
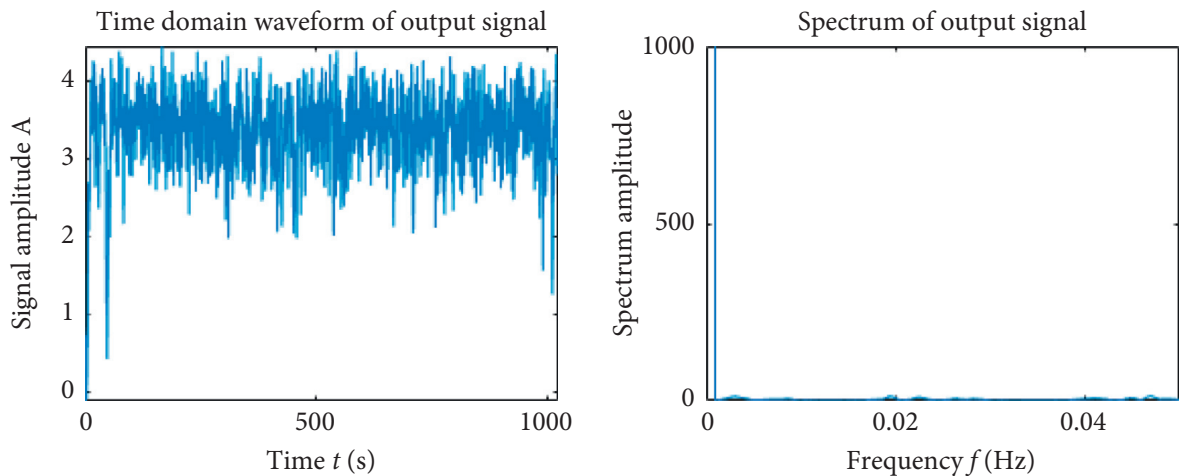

(a)
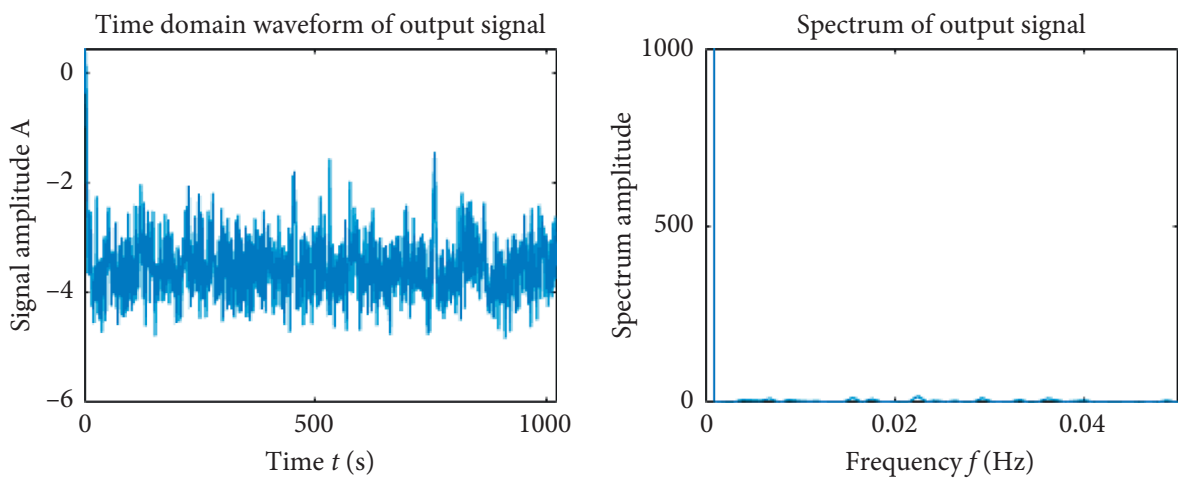

(b)

Figure 5: Resonance results of the original public opinion resonance mode. (a) Original model (Chongqing vaccine event-Shandong vaccine event). (b) Original model (Changsheng vaccine event-Shandong vaccine event).

emergencies directly affects the generation of public opinion resonance phenomenon. The higher the topical relevance degree, the more obvious the phenomenon of public opinion resonance between events. So, there is a positive correlation between them.

Therefore, according to the above verification results, in future public health emergencies or other subject areas of network public opinion monitoring and early warning tasks, the relevant storage data in the historical public opinion event database can be calculated, and the simulation can be performed through the model. Predict and judge the possibility that historical public opinion events will be "resurgence" or resonate with recent events before the incident and rely on the possibility to eliminate hidden dangers in advance and make timely response strategies. At the same time, it is necessary to pay special attention to checking and excluding events with high thematic similarity, because these events are more likely to be induced. 


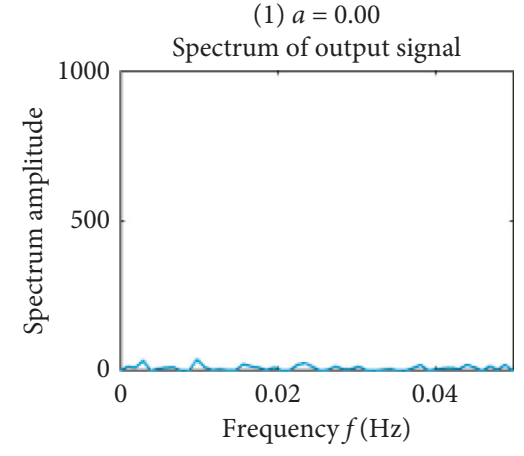

(4) $a=0.60$

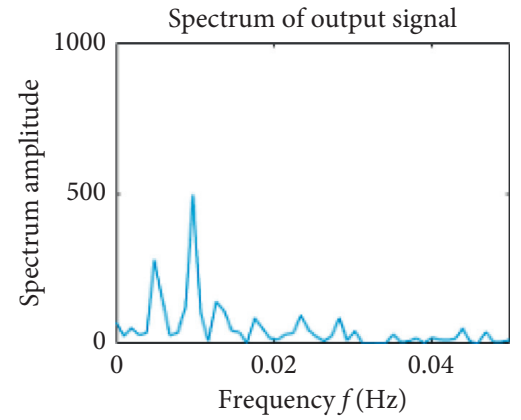

(1) $a=0.00$

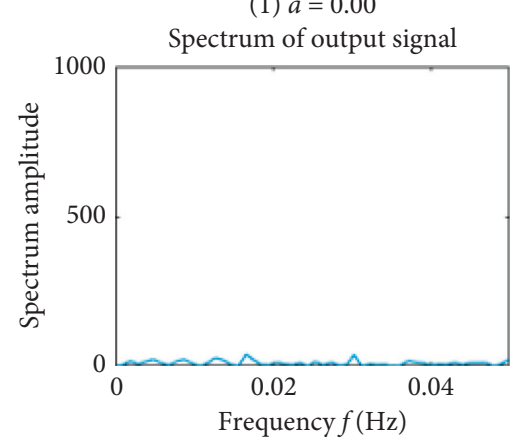

(4) $a=0.60$

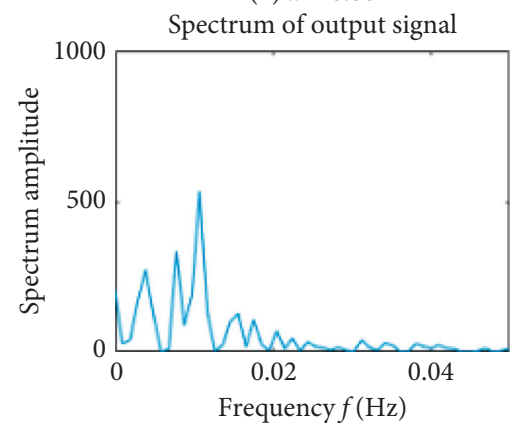

(2) $a=0.20$

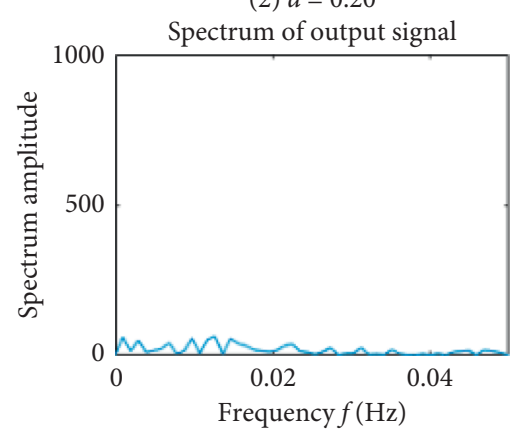

(5) $a=0.80$

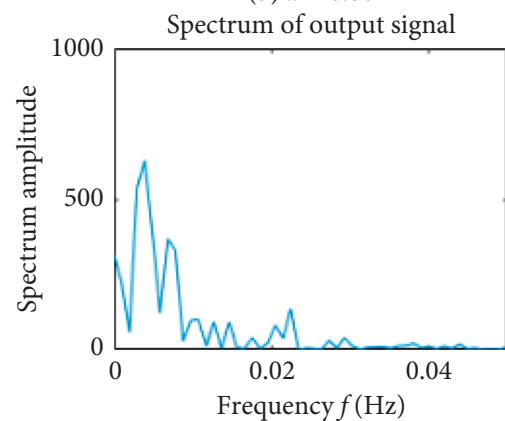

(a)

(2) $a=0.20$

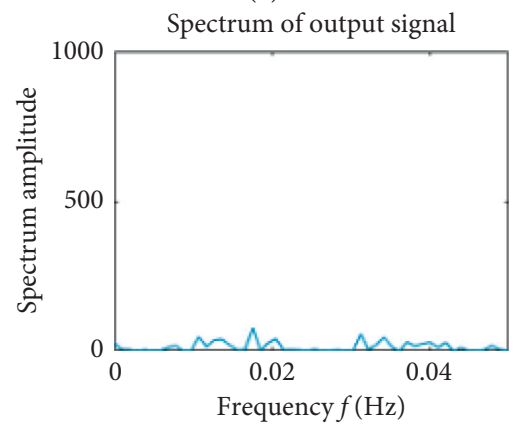

(5) $a=0.80$

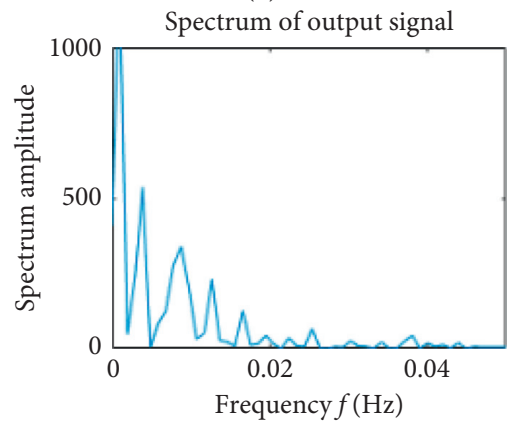

(b)

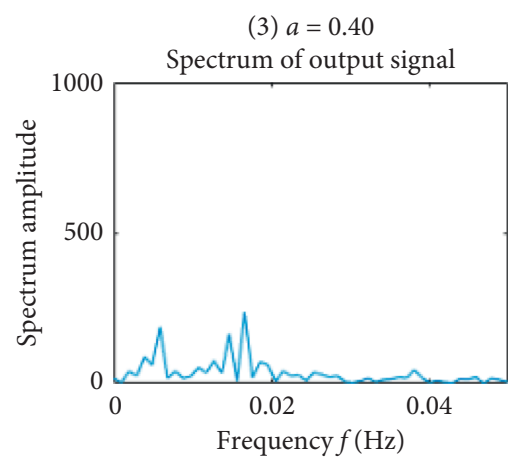

(6) $a=1.00$

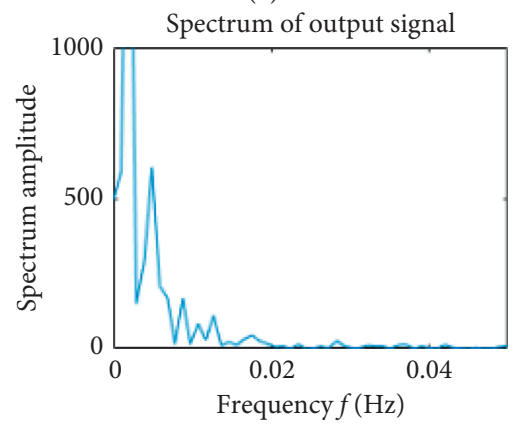

(3) $a=0.40$

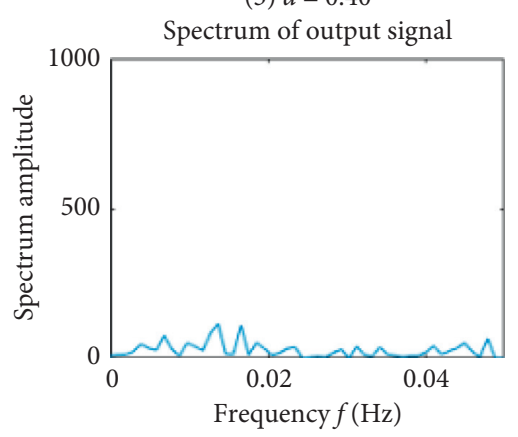

(6) $a=1.00$

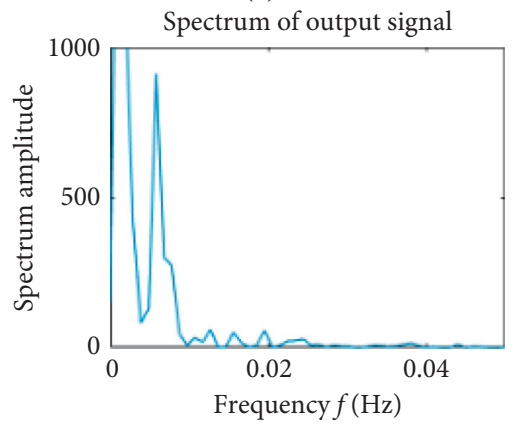

FIGURE 6: The influence of the topic-related factors on public opinion resonance. (a) Chongqing vaccine event-Shandong vaccine event. (b) Changsheng vaccine event-Shandong vaccine event.

\section{Conclusions}

This paper studies the resonance phenomenon and regulations of Internet public opinion caused by the frequent public health emergencies; at the same time, the influencing factors of the topic relevance of Internet public opinion resonance are experimentally verified. For this reason, we analyze the influence factors of the Internet public opinion events in the public health emergencies and propose the Internet public opinion stochastic resonance model 
considering the topic relevance based on Langevin's equation, meanwhile, providing three exact public health emergencies in China to reveal the regulations of evoked events "revival" caused by original events. Through the above research and simulation experiments, we obtain the following results:

(1) The Internet public opinion resonance model considering the topic relevance has significantly improved in terms of sensitivity, significance, resonance effect, and the degree of influence of topical relevance on resonance.

(2) The model has more significant advantages in the resonance phenomenon and regulation disclosure of public opinion between multiple public health emergencies due to the theme relevance, interaction, and interactivity.

(3) There is a significant positive correlation between the resonance degree of public opinion in public health emergencies and the time interval between events and the topic relevance degree.

These results not only provides a feasible idea for the study of multidimensional prediction of Internet public opinion in public health emergencies but also plays an important role in supporting the public health management departments in the aspects of topic division, classification management, identification of response information needs, and making management decisions. This study still has its limitations; a single source of data (Sina MicroBlog) leads to lack of comprehensiveness; the selection of study cases makes the results of resonance simulation have randomness. In future research, it is proposed to use multiplatform and multievent relatively comprehensive data to analyze the phenomenon and regulation of Internet public opinion resonance between multidomain topic-related emergencies.

\section{Data Availability}

The data used to support this study are available from the corresponding author on reasonable request.

\section{Conflicts of Interest}

The authors declare that they have no conflicts of interest.

\section{Acknowledgments}

This work was partly supported by the Social Science Fund of China under grant no. 19BTQ072.

\section{References}

[1] O. Edo-Osagie, B. De La Iglesia, I. Lake, and O. Edeghere, "A scoping review of the use of Twitter for public health research," Computers in Biology and Medicine, vol. 122, Article ID 103770, 2020.

[2] H. Sánchez, J. Aguilar, O. Terán, and J. Gutiérrez de Mesa, "Modeling the process of shaping the public opinion through multilevel fuzzy cognitive maps," Applied Soft Computing, vol. 85, Article ID 105756, 2019.
[3] H. Zhu and B. Hu, "Impact of information on public opinion reversal-an agent based model," Physica A: Statistical Mechanics and Its Applications, vol. 512, pp. 578-587, 2018.

[4] S. Li, Z. Liu, and Y. Li, "Temporal and spatial evolution of online public sentiment on emergencies," Information Processing \& Management, vol. 57, no. 2, Article ID 102177, 2020.

[5] G. Wang, Y. Chi, Y. Liu, and Y. Wang, "Studies on a multidimensional public opinion network model and its topic detection algorithm," Information Processing \& Management, vol. 56, no. 3, pp. 584-608, 2019.

[6] L. Ismailova, V. Wolfengagen, S. Kosikov, M. Maslov, and J. Dohrn, "Semantic models to indicate post-truth with fake news channels," Procedia Computer Science, vol. 169, pp. 297-303, 2020.

[7] W. WuGoh, M. Wang, and Y.-c. Zhu, "Does government information release really matter in regulating contagionevolution of negative emotion during public emergencies? From the perspective of cognitive big data analytics," International Journal of Information Management, vol. 50, pp. $498-514,2020$.

[8] Y. X. Lan, X. L. Dong, and R. X. Zeng, "Research on effects model of derivative of network public opinion from the perspective of information alienation," Journal of Intelligence, vol. 34, no. 1, pp. 139-143, 2015.

[9] Z. X. Lian, Z. S. Lian, and Q. B. Zhang, "Derived network early warning model of public opinion and its empirical research," Journal of Intelligence, vol. 38, no. 3, pp. 133-140, 2019.

[10] L. Zhang, C. Su, Y. Jin, M. Goh, and Z. Wu, "Cross-network dissemination model of public opinion in coupled networks," Information Sciences, vol. 451-452, pp. 240-252, 2018.

[11] R. Benzi, A. Sutera, and A. Vulpiani, "The mechanism of stochastic resonance," Journal of Physics A, vol. 14, pp. L453-L457, 1981.

[12] L. J. Wang and J. H. Dai, "Research on the quantitative analytical method of network public opinion derivative chain," Information Science, vol. 34, no. 7, pp. 59-63, 2016.

[13] B. Gao and L. C. Wang, "Propagation model and analysis method of network-derived public opinion," Information Studies: Theorye Application, vol. 42, no. 3, pp. 166-170, 2019.

[14] Y. Xie, R. Qiao, G. Shao, and H. Chen, "Research on Chinese social media users' communication behaviors during public emergency events," Telematics and Informatics, vol. 34, no. 3, pp. 740-754, 2017.

[15] H. Q. Li, "Research on evolution law and countermeasure of the derived network public opinion based on information alienation theory-taking internet rumors governance for example," Journal of Modern Information, vol. 35, no. 5, pp. 4-8, 2015.

[16] C. Li, J. Bai, L. Zhang, H. Tang, and Y. Luo, "Opinion community detection and opinion leader detection based on text information and network topology in cloud environment," Information Sciences, vol. 504, pp. 61-83, 2019.

[17] N. Ma and Y. Liu, "SuperedgeRank algorithm and its application in identifying opinion leader of online public opinion super network," Expert Systems with Applications, vol. 41, no. 4, pp. 1357-1368, 2014.

[18] X. Dong, Y. Lian, X. Tang, and Y. Liu, “The damped oscillator model (DOM) and its application in the prediction of emotion development of online public opinions," Expert Systems with Applications, vol. 148, Article ID 113268, 2020.

[19] X. Liu, W. Xu, and B. Li, "Reason and passion in public discussion on Sina Weibo," Telematics and Informatics, vol. 45, Article ID 101285, 2019. 
[20] Y. N. Li, Research on Resonance Phenomenon of Public Opinion Based on Stochastic Resonance Model, China University of Geosciences, Wuhan, China, 2018.

[21] M. N. Chen, "Research on the naming mechanism of news events in the era public sentiment 2.0," Contemporary Communication, vol. 6, pp. 52-54, 2019.

[22] D. Liu, "Particle-scale modelling of financial price dynamics," Communications in Nonlinear Science and Numerical Simulation, vol. 43, pp. 282-295, 2017.

[23] N. A. Schenck, P. A. Horvath, and A. K. Sinha, "Understanding price discovery in interconnected markets: generalized Langevin process approach and simulation," Physica A: Statistical Mechanics and Its Applications, vol. 491, pp. 741748, 2018. 